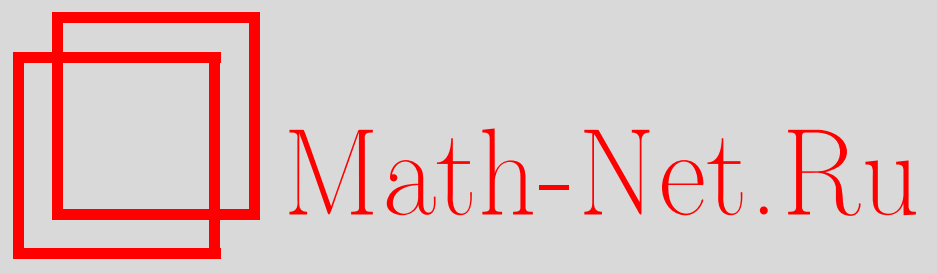

Т. В. Волкова, М. В. Долгополов, М. Н. Дубинин, Э. Н. Рыкова, Эффективный потенциал Хиггса в неминимальной суперсимметричной Стандартной модели, Вестн. Сам. гос. техн. ун-та. Сер. Физ.-мат. науки, 2013, выпуск 2(), 233-242

DOI: https://doi.org/10.14498/vsgtu1193

Использование Общероссийского математического портала Math-Net.Ru подразумевает, что вы прочитали и согласны с пользовательским соглашением

http: //www . mathnet.ru/rus/agreement

Параметры загрузки:

IP : 54.224 .187 .69

26 апреля 2023 г., 06:58:26

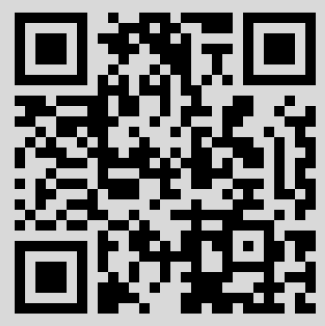




\title{
ЭФФЕКТИВНЫЙ ПОТЕНЦИАЛ ХИГГСА В НЕМИНИМАЛЬНОЙ СУПЕРСИММЕТРИЧНОЙ СТАНДАРТНОЙ МОДЕЛИ
}

\author{
Т. В. Волкова ${ }^{1}$, М. В. Долгополов ${ }^{1}$, М. Н. Дубинин ${ }^{2}$, Э. Н. Рыкова \\ 1 Самарский государственный университет, \\ Россия, 443011, Самара, ул. Академика Павлова, 1. \\ 2 Научно-исследовательский институт ядерной физики им. Д. В. Скобельцына, \\ Московский государственный университет им. М. В. Ломоносова, \\ Россия, 119991, Москва, Ленинские горы. \\ E-mails: milandiya@yandex.ru, mikhaildolgopolov@rambler.ru, \\ dubinin@theory.sinp.msu.ru, elzarykova@rambler.ru
}

\begin{abstract}
Представлен хиггсовский сектор неминимальной суперсимметричной стандартной модели с нарушением СР-инвариантности в суперпотенииале и в секторе мягкого нарушения суперсимметрии. Рассмотрены однопетлевые поправки $\kappa$ параметрам эфбективного потенииала и получены значения масс нейтральных бозонов Хиггса.
\end{abstract}

Ключевые слова: сектор Хиггса, неминимальная суперсимметричная стандартная модель, массы бозонов Хиггса, эффективный потенииал Хиггса.

Введение. Хорошо известно, что неминимальная суперсимметричная стандартная модель (HМCCM) [1-8] обеспечивает решение так называемой « $\mu$-проблемы» [9] минимальной суперсимметричной стандартной модели (MCCM) с помощью введения дополнительного синглетного суперполя $S$. Вопрос о порядке величины параметра $\mu$ хиггсовского суперпотенциала

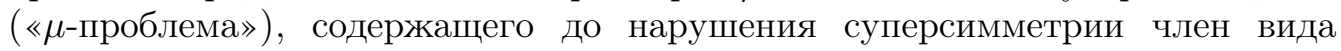
$\mu H_{1} H_{2}$, оправданно возникает в MCCM, поскольку естественные значения для параметра $\mu$-либо нуль, либо планковский масштаб. Первое весьма нежелательно, так как приводит к ненаблюдаемому экспериментально аксиону, когда электрослабая симметрия спонтанно нарушается, а второе не представляет интереса, поскольку планковский масштаб параметра $\mu$ воспроизводит известную проблему калибровочных иерархий. В связи с необходимостью исключения явно нарушающего $C P$-инвариантность сильных взаимодействий $\theta$-члена квантовой хромодинамики есть основания полагать, что $\mu$ имеет порядок $\mathcal{O}\left(M_{W}\right)$ или немного более, хотя для этого нужно расширить хиггсовский сектор для получения невидимого аксиона [9-17]. В чём причина того, что величина $\mu$ должна быть на 15-17 порядков меньше по сравнению с массой Планка, и есть основное содержание « $\mu$-проблемы» МССМ. Также относительно малое значение $\mu$ приводит к «собственной» проблеме иерархии MCCM, состоящей в большом отличии параметра $\mu$ от масштаба нарушения суперсимметрии $M_{\mathrm{SUSY}}$. Условия для электрослабого нарушения симметрии требуют, чтобы значение $\mu$ было порядка массы $Z$-бозона. Кроме того, МССМ имеет ряд ограничений на область параметров, совместную с исследования-

Татълна Васильевна Волкова, магистрант, лаб. математической физики. Михаил Вячеславович Долгополов (к.ф-м.н., доц.), доцент, каф. общей и теоретической физики. Михаил Николаевич Дубинин (д.ф-м.н.), ведущий научный сотрудник, отдел теоретической физики высоких энергий. Элъза Нуровна Рыкова (к.ф-м.н.), старший преподаватель, каф. общей и теоретической физики. 
ми на LHC, что требует рассмотрения эффективных сценариев за рамками MCCM.

В общем случае решение « струнных $E_{6}$ моделях, где билинейные члены суперпотенциала запрещены калибровочной симметрией. Такие модели содержат несколько пар дублетов Хиггса и несколько синглетных полей $S_{i}$. Если при низких энергиях эффективно остаются только одна пара дублетов Хиггса и один синглет, то сектор Хиггса содержит член $\lambda \hat{S}\left(\hat{H}_{1} \epsilon \hat{H}_{2}\right)$. Подобная модель включает только одно добавочное синглетное суперполе и почти то же количество параметров, что и МССМ. Поэтому в этом смысле она является простейшим расширением MCCM. В результате спонтанного нарушения симметрии на электрослабом масштабе суперполе $\hat{S}$ приобретает ненулевую величину вакуумного ожидания $(\langle S\rangle \equiv s / \sqrt{2})$ и генерируется требуемый эффективный $\mu$-член $\mu H_{1} H_{2}$, где $\mu=\lambda s / \sqrt{2}$.

Модели упомянутого типа имеют глобальную симметрию $S U(2) \times[U(1)]^{2}$. Как уже упоминалось, спонтанное нарушение расширенной глобальной симметрии приводит к появлению безмассовой $C P$-нечётной скалярной частицы-PQ-аксиона [18]. Его можно исключить, если ввести в суперпотенциал кубический по новому синглетному суперполю $\hat{S}$ член, явно нарушающий добавочную глобальную симметрию $U(1)$. Тогда суперпотенциал сектора Хиггса модели НМССМ имеет вид

$$
W_{H}=\lambda \hat{S}\left(\hat{H}_{1} \epsilon \hat{H}_{2}\right)+\frac{1}{3} \kappa \hat{S}^{3} .
$$

Эффективный $\mu$-член, возникающий при вакуумном среднем $S$ порядка масштаба нарушения суперсимметрии, будет иметь порядок электрослабого, если масштаб нарушения суперсимметрии $M_{\text {SUSY }}$ не очень велик по сравнению с массой $W$-бозона. В этом смысле НМССМ есть простейшее суперсимметричное расширение Стандартной модели, в котором электрослабая шкала возникает исключительно из шкалы нарушения суперсимметрии. В рамках НМССМ более естественно (с точки зрения подбора параметров) выглядит ненаблюдаемость лёгкого нейтрального $C P$-чётного бозона Хиггса на LEP2 [19]. Хотя симметрии НMCСМ могут привести к возможности проблемы космологической доменной стенки [20], этого можно избежать введением подходящих ненормируемых операторов [21-26], которые не дают большие вклады синглетных диаграмм-головастиков (tadpole diagrams). Эти дополнительные операторы могут быть выбраны достаточно малыми для того, чтобы не изменить низкоэнергетическую феноменологию.

В дополнение к полям МССМ НMCСМ содержит еще $C P$-чётный и $C P$ нечётный бозоны Хиггса, а также еще одно нейтралино. Новые поля смешиваются с соответствующими полями MCCM, приводя к более интересной и сложной феноменологии: возможности существования очень лёгкого нейтралино [27] как кандидата на роль частиц темной материи, увеличения ограничения сверху на массу легчайшего бозона Хиггса по сравнению с МССМ [28] и возможности существования очень лёгкого бозона Хиггса $[29,30]$ или невидимого бозона Хиггса. Эти свойства НМССМ могут существенно модифицировать известные предсказания MCCM для экспериментов на LHC.

В данной работе исследуется эффективный хиггсовский потенциал 
НМССМ в случае, когда его $C P$-инвариантность явно нарушена. Проводится диагонализация массового члена этого потенциала в локальном минимуме. Вычислены однопетлевые поправки к параметрам эффективного потенциала, обусловленные взаимодействиями полей Хиггса с третьим поколением скалярных кварков, а также с суперпартнерами калибровочного сектора, получены физические состояния бозонов Хиггса и их массы.

1. Структура сектора Хиггса НМССМ. Рассмотрим общую модель хиггсовского сектора с двумя дублетами комплексных полей $\Phi_{1}, \Phi_{2}$ и одним синглетом поля $S$ :

$$
\begin{gathered}
\Phi_{1}=\left(\begin{array}{c}
\phi_{1}^{+}(x) \\
\phi_{1}^{0}(x)
\end{array}\right)=\left(\begin{array}{c}
-i \omega_{1}^{+} \\
\frac{1}{\sqrt{2}}\left(v_{1}+\eta_{1}+i \chi_{1}\right)
\end{array}\right), \\
\Phi_{2}=\left(\begin{array}{c}
\phi_{2}^{+}(x) \\
\phi_{2}^{0}(x)
\end{array}\right)=\left(\begin{array}{c}
-i \omega_{2}^{+} \\
\frac{1}{\sqrt{2}}\left(v_{2}+\eta_{2}+i \chi_{2}\right)
\end{array}\right), \\
S=\frac{1}{\sqrt{2}}\left(v_{3}+s_{1}+i s_{2}\right) .
\end{gathered}
$$

Наиболее общая калибровочно-инвариантная перенормируемая форма потенциала имеет следующий вид:

$$
\begin{gathered}
U\left(\Phi_{1}, \Phi_{2}, S\right)=-\mu_{1}^{2}\left(\Phi_{1}^{\dagger} \Phi_{1}\right)-\mu_{2}^{2}\left(\Phi_{2}^{\dagger} \Phi_{2}\right)-\mu_{3}^{2}\left(S^{\dagger} S\right)+ \\
+\frac{\lambda_{1}}{2}\left(\Phi_{1}^{\dagger} \Phi_{1}\right)^{2}+\frac{\lambda_{2}}{2}\left(\Phi_{2}^{\dagger} \Phi_{2}\right)^{2}+\lambda_{3}\left(\Phi_{1}^{\dagger} \Phi_{1}\right)\left(\Phi_{2}^{\dagger} \Phi_{2}\right)+\lambda_{4}\left(\Phi_{1}^{\dagger} \Phi_{2}\right)\left(\Phi_{2}^{\dagger} \Phi_{1}\right)+ \\
+\frac{\lambda_{5}}{2}\left(\Phi_{1}^{\dagger} \Phi_{2}\right)\left(\Phi_{1}^{\dagger} \Phi_{2}\right)+\frac{\lambda_{5}^{*}}{2}\left(\Phi_{2}^{\dagger} \Phi_{1}\right)\left(\Phi_{2}^{\dagger} \Phi_{1}\right)+ \\
+\lambda_{6}\left(\Phi_{1}^{\dagger} \Phi_{2}\right)\left(\Phi_{1}^{\dagger} \Phi_{1}\right)+\lambda_{6}^{*}\left(\Phi_{2}^{\dagger} \Phi_{1}\right)\left(\Phi_{1}^{\dagger} \Phi_{1}\right)+\lambda_{7}\left(\Phi_{1}^{\dagger} \Phi_{2}\right)\left(\Phi_{2}^{\dagger} \Phi_{2}\right)+\lambda_{7}^{*}\left(\Phi_{2}^{\dagger} \Phi_{1}\right)\left(\Phi_{2}^{\dagger} \Phi_{2}\right)+ \\
+k_{1}\left(\Phi_{1}^{\dagger} \Phi_{1}\right)\left(S^{\dagger} S\right)+k_{2}\left(\Phi_{2}^{\dagger} \Phi_{2}\right)\left(S^{\dagger} S\right)+k_{3}\left(\Phi_{1}^{\dagger} \Phi_{2}\right)\left(S^{\dagger} S^{\dagger}\right)+k_{3}\left(\Phi_{2}^{\dagger} \Phi_{1}\right)(S S)+ \\
+k_{4}\left(S^{\dagger} S\right)^{2}+k_{5}\left(\Phi_{1}^{\dagger} \Phi_{2}\right) S+k_{5}\left(\Phi_{2}^{\dagger} \Phi_{1}\right) S^{\dagger}+k_{6} S^{3}+k_{6}\left(S^{\dagger}\right)^{3} .
\end{gathered}
$$

Потенциал инвариантен относительно группы $S U(2) \times U(1) \times Z_{3}$, при этом поля бозонов Хиггса относительно $Z_{3}$ преобразуются следующим образом:

$$
\Phi_{1} \rightarrow \exp [i(2 m \pi / 3)] \Phi_{1}, \quad \Phi_{2} \rightarrow \Phi_{2}, \quad S \rightarrow \exp [i(2 n \pi / 3)] S,
$$

где $m$ и $n$ - целые числа (в частности, $(m, n)=(1,1)$ или $(2,2))$.

Вакуумные ожидания хиггсовских дублетов и синглета выберем в виде

$$
\left\langle\Phi_{1}\right\rangle=\frac{1}{\sqrt{2}}\left(\begin{array}{c}
0 \\
v_{1}
\end{array}\right), \quad\left\langle\Phi_{2}\right\rangle=\frac{1}{\sqrt{2}}\left(\begin{array}{c}
0 \\
v_{2}
\end{array}\right), \quad\langle S\rangle=\frac{1}{\sqrt{2}} v_{3} .
$$

В древесном приближении на масштабе энергий нарушения суперсимметрии параметры эффективного потенциала $\lambda_{i}$ являются действительными и 
выражаются граничными условиями через константы связи $g_{1}$ и $g_{2}$ электрослабой группы калибровочной симметрии $S U(2) \times U(1)$ :

$$
\begin{gathered}
\lambda_{1}\left(M_{\mathrm{SUSY}}\right)=\lambda_{2}\left(M_{\mathrm{SUSY}}\right)=\frac{1}{4}\left(g_{2}^{2}\left(M_{\mathrm{SUSY}}\right)+g_{1}^{2}\left(M_{\mathrm{SUSY}}\right)\right), \\
\lambda_{3}\left(M_{\mathrm{SUSY}}\right)=\frac{1}{4}\left(g_{2}^{2}\left(M_{\mathrm{SUSY}}\right)-g_{1}^{2}\left(M_{\mathrm{SUSY}}\right)\right), \quad \lambda_{4}\left(M_{\mathrm{SUSY}}\right)=-\frac{1}{2} g_{2}^{2}\left(M_{\mathrm{SUSY}}\right), \\
\lambda_{5}\left(M_{\mathrm{SUSY}}\right)=\lambda_{6}\left(M_{\mathrm{SUSY}}\right)=\lambda_{7}\left(M_{\mathrm{SUSY}}\right)=0 .
\end{gathered}
$$

Параметры $k_{i}$ определяются следующим образом: $k_{1}=|\lambda|^{2}, k_{2}=|\lambda|^{2}$, $k_{3}=\lambda k^{*}, k_{4}=|k|^{2}, k_{5}=\lambda A_{\lambda}, k_{6}=k A_{k} / 3$, что соответствует обозначениям работы [31].

2. Однопетлевые поправки к параметрам эффективного потенциала. В данной статье применяется метод, развитый авторами в работах [32-35]. Вычисления проводились диаграммным способом с использованием потенциала мягкого нарушения суперсимметрии, включающего взаимодействия бозонов Хиггса с третьим поколением суперпартнеров кварков. В рамках этого подхода радиационные поправки к параметрам эффективного потенциала $\lambda_{1}, \lambda_{2}, \ldots, \lambda_{7}$ и $k_{1}, k_{2} \ldots, k_{6}$ получены путём явного расчёта однопетлевых диаграмм с четырьмя внешними линиями. При этом параметры $\lambda_{1}, \lambda_{2}, \ldots, \lambda_{7}$ и $k_{1}, k_{2} \ldots, k_{6}$ аналитически выражаются через параметры взаимодействия сектора «скалярные кварки - бозоны Хиггса».

Члены суперсимметричного скалярного потенциала, содержащие члены взаимодействия бозонов Хиггса со скалярными кварками третьего поколения, определяются следующим образом [36]:

$$
\begin{array}{r}
V \supset\left|h_{u}\left(\tilde{Q} \epsilon \Phi_{1}\right)\right|^{2}+\left|h_{d}\left(\tilde{Q} \epsilon \Phi_{2}\right)\right|^{2}+\left|h_{u} \tilde{u}_{R}^{*} \Phi_{1}^{0}\right|^{2}+\left|h_{d} \tilde{d}_{R}^{*} \Phi_{2}^{0}\right|^{2}+\left|h_{u} \tilde{u}_{R}^{*} \tilde{u}_{L}-\lambda S \Phi_{2}^{0}\right|^{2}+ \\
+\left|h_{d} \tilde{d}_{R}^{*} \tilde{d}_{L}-\lambda S \Phi_{1}^{0}\right|^{2}+4\left|\Phi_{2}^{\dagger} \tilde{Q}\right|^{2}-2\left(\Phi_{2}^{\dagger} \Phi_{2}\right)\left(\tilde{Q}^{\dagger} \tilde{Q}\right)+4 \mid \Phi_{1}^{\dagger} \tilde{Q}^{2}-2\left(\Phi_{1}^{\dagger} \Phi_{1}\right)\left(\tilde{Q}^{\dagger} \tilde{Q}\right)+ \\
+\frac{g_{1}^{2}}{2}\left(\frac{1}{6} \tilde{Q}^{\dagger} \tilde{Q}-\frac{2}{3} \tilde{u}_{R}^{*} \tilde{u}_{R}+\frac{1}{3} \tilde{d}_{R}^{*} \tilde{d}_{R}+\frac{1}{2} \Phi_{1}^{\dagger} \Phi_{1}-\frac{1}{2} \Phi_{2}^{\dagger} \Phi_{2}\right)^{2}+ \\
+\left(\tilde{u}_{R}^{*} h_{u} A_{u}\left(\tilde{Q}^{\top} \epsilon \Phi_{1}\right)-\tilde{d}_{R}^{*} h_{d} A_{d}\left(\tilde{Q}^{\top} \epsilon \Phi_{2}\right)+\text { э.c. }\right) .
\end{array}
$$

В результате вычислений получаем следующие аналитические выражения для поправок:

$$
\begin{gathered}
\Delta \lambda_{1}=h_{u}^{4} A_{u}^{4} I_{2}\left(m_{U}, m_{Q}\right)+h_{u}^{4} A_{u}^{2} I_{1}\left(m_{U}, m_{Q}\right)+h_{u}^{4} A_{u}^{2} I_{1}\left(m_{Q}, m_{U}\right)- \\
-\frac{g_{1}^{2}}{3} h_{u}^{2} A_{u}^{2} I_{1}\left(m_{U}, m_{Q}\right)+\left(\frac{g_{1}^{2}}{12}-\frac{g_{2}^{2}}{4}\right) h_{u}^{2} A_{u}^{2} I_{1}\left(m_{Q}, m_{U}\right), \\
\Delta \lambda_{2}=h_{d}^{4} A_{d}^{4} I_{2}\left(m_{D}, m_{Q}\right)+h_{d}^{4} A_{d}^{2} I_{1}\left(m_{D}, m_{Q}\right)+h_{d}^{4} A_{d}^{2} I_{1}\left(m_{Q}, m_{D}\right)- \\
-\frac{g_{1}^{2}}{6} h_{d}^{2} A_{d}^{2} I_{1}\left(m_{D}, m_{Q}\right)+\left(-\frac{g_{1}^{2}}{12}-\frac{g_{2}^{2}}{4}\right) h_{d}^{2} A_{d}^{2} I_{1}\left(m_{Q}, m_{D}\right),
\end{gathered}
$$

$$
\Delta k_{1}=\lambda^{2} h_{d}^{2} I\left(m_{Q}, m_{D}\right), \quad \Delta k_{2}=\lambda^{2} h_{u}^{2} I\left(m_{Q}, m_{U}\right)
$$




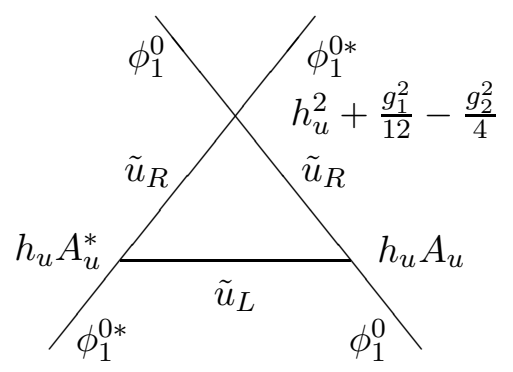

$$
\left(h_{u}^{2}+\frac{g_{1}^{2}}{12}-\frac{g_{2}^{2}}{4}\right) h_{u}^{2}\left|A_{u}\right|^{2} I_{1}\left(m_{U}, m_{Q}\right)
$$

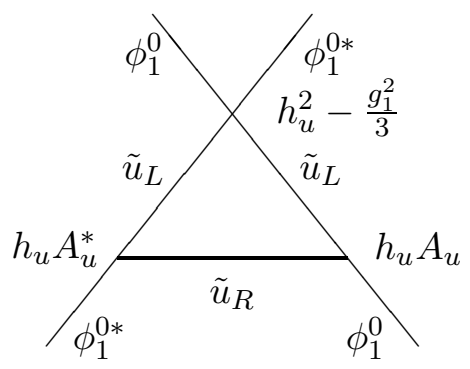

$$
\left(h_{u}^{2}-\frac{g_{1}^{2}}{3}\right) h_{u}^{2}\left|A_{u}\right|^{2} I_{1}\left(m_{Q}, m_{U}\right)
$$

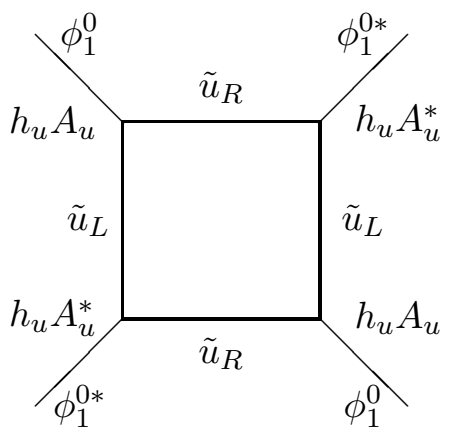

$$
h_{u}^{4}\left|A_{u}\right|^{4} I_{2}\left(m_{Q}, m_{U}\right)
$$

Однопетлевые диаграммы Фейнмана и поправки в параметр $\lambda_{1}$ эффективного потенциала

Интегралы $I_{i}$ были рассчитаны ранее в работах [35, 37-39]. Для случая разных масс скалярных кварков получаем

$$
\begin{gathered}
I\left(m_{1}, m_{2}\right)=\int \frac{d^{4} k}{(2 \pi)^{4}} \frac{i}{\left(k^{2}-m_{1}^{2}\right)\left(k^{2}-m_{2}^{2}\right)} \equiv \frac{1}{16 \pi^{2}} B_{0}\left(m_{1}^{2}, m_{2}^{2}\right)= \\
=\frac{1}{16 \pi^{2}} \log \left(\frac{m_{1}^{2}}{m_{t}^{2}}\right)-\frac{m_{2}^{2}}{m_{1}^{2}-m_{2}^{2}} \log \left(\frac{m_{2}^{2}}{m_{1}^{2}}\right), \\
I_{1}\left(m_{1}, m_{2}\right)=\int \frac{d^{4} k}{(2 \pi)^{4}} \frac{i}{\left(k^{2}-m_{1}^{2}\right)^{2}\left(k^{2}-m_{2}^{2}\right)} \equiv \frac{1}{16 \pi^{2}} C_{0}\left(m_{2}^{2}, m_{1}^{2}\right)= \\
=\frac{1}{m_{1}^{2}-m_{2}^{2}}\left(1+\frac{m_{2}^{2}}{m_{1}^{2}-m_{2}^{2}} \log \left(\frac{m_{2}^{2}}{m_{1}^{2}}\right)\right),
\end{gathered}
$$




$$
\begin{gathered}
I_{2}\left(m_{1}, m_{2}\right)=\int \frac{d^{4} k}{(2 \pi)^{4}} \frac{i}{\left(k^{2}-m_{1}^{2}\right)^{2}\left(k^{2}-m_{2}^{2}\right)^{2}} \equiv \frac{1}{16 \pi^{2}} D_{0}\left(m_{1}^{2}, m_{2}^{2}\right)= \\
=\frac{2}{\left(m_{1}^{2}-m_{2}^{2}\right)^{2}}+\frac{m_{1}^{2}+m_{2}^{2}}{\left(m_{2}^{2}-m_{1}^{2}\right)^{3}} \log \left(\frac{m_{1}^{2}}{m_{2}^{2}}\right) .
\end{gathered}
$$

3. Массовые состояния бозонов Хиггса в НМССМ. Для получения физических состояний бозонов Хиггса необходимо выполнение условий существования локального минимума потенциала $U$ в пространстве $\left(v_{1}, v_{2}, v_{3}\right)$ :

$$
\begin{aligned}
& \mu_{1}^{2}=\frac{1}{2}\left(v^{2} \lambda_{1} \cos ^{2} \beta\right.+v^{2}\left(\lambda_{3}+\lambda_{4}+\operatorname{Re} \lambda_{5}\right) \sin \beta^{2}+ \\
&\left.+v^{2} \sin \beta\left(3 \operatorname{Re} \lambda_{6} \cos \beta+\operatorname{Re} \lambda_{7} \tan \beta\right)\right)+ \\
&+\frac{1}{2} k_{1} v_{3}^{2}+\left(\frac{1}{2} \operatorname{Re} k_{3} v_{3}+\frac{1}{\sqrt{2}} \operatorname{Re} k_{5}\right) v_{3} \tan \beta, \\
&+\frac{1}{2} k_{2} v_{3}^{2}+\left(\frac{1}{2} \operatorname{Re} k_{3} v_{3}+\frac{1}{\sqrt{2}} \operatorname{Re} k_{5}\right) v_{3} \cot \beta, \\
&\left.+v^{2} \cos \beta\left(3 \operatorname{Re} \lambda_{7} \sin \beta+\operatorname{Re} \lambda_{6} \cot \beta\right)\right)+ \\
&+v^{2}\left(\lambda_{3}+\lambda_{4}^{2} \lambda_{2} \sin ^{2} \beta\right. \\
& \mu_{3}^{2}=\frac{v^{2}}{2}\left(k_{1} \cos ^{2} \beta\right.\left.+k_{2} \sin ^{2} \beta+\left(\operatorname{Re} k_{3}+\frac{1}{\sqrt{2} v_{3}} \operatorname{Re} k_{5}\right) \sin 2 \beta\right)+ \\
&+\operatorname{Re} k_{4} v_{3}^{2}+\frac{3}{2} \operatorname{Re} k_{6} v_{3},
\end{aligned}
$$

где $v^{2}=v_{1}^{2}+v_{2}^{2}, \tan \beta=v_{2} / v_{1}$. Они обеспечивают обращение в нуль линейных по полям членов $\eta_{1}, \eta_{2}$.

В НМССМ симметрическая массовая матрица для нейтральных бозонов Хиггса в базисе $\left(\eta_{1}, \eta_{2}, s_{1}, A, s_{2}\right)$ с $A=-\chi_{1} \sin \beta+\chi_{2} \cos \beta$ имеет сложную структуру. В случае нарушения $C P$-инвариантности необходимо рассматривать всю $5 \times 5$-матрицу, для которой собственные состояния не будут обладать определенной $C P$-чётностью.

Рассмотрим базис $\left(H, A, h, s_{1}, s_{2}\right)$ с $h=-\eta_{1} \sin \beta+\eta_{2} \cos \beta$ и $H=\eta_{1} \cos \beta+$ $+\eta_{2} \sin \beta$, в котором симметрическая массовая $5 \times 5$-матрица имеет вид

$$
M^{2}=\left(\begin{array}{ccccc}
m_{11} & m_{12} & m_{13} & m_{14} & m_{15} \\
m_{21} & m_{22} & m_{23} & m_{24} & m_{25} \\
m_{31} & m_{32} & m_{33} & m_{34} & m_{35} \\
m_{41} & m_{42} & m_{43} & m_{44} & m_{45} \\
m_{51} & m_{52} & m_{53} & m_{54} & m_{55}
\end{array}\right)
$$

где

$$
\begin{aligned}
m_{11}=\frac{v^{2}}{2}\left(\lambda_{1} \cos ^{4} \beta+\lambda_{2} \sin ^{4} \beta+\right. & 2\left(\lambda_{3}+\lambda_{4}+\operatorname{Re} \lambda_{5}\right) \cos ^{2} \beta \sin ^{2} \beta+ \\
& \left.+4 \operatorname{Re} \lambda_{6} \cos ^{3} \beta \sin \beta+4 \operatorname{Re} \lambda_{7} \cos \beta \sin ^{3} \beta\right),
\end{aligned}
$$




$$
\begin{aligned}
& m_{12}=\frac{1}{4}\left(3 v^{2}\left(\operatorname{Im} \lambda_{5} \sin 2 \beta+\operatorname{Im} \lambda_{6}(1+\cos 2 \beta)+\operatorname{Im} \lambda_{7}(1-\cos 2 \beta)\right)-\right. \\
& \left.-2 v_{3}\left(v_{3} \operatorname{Im} k_{3}+\sqrt{2} \operatorname{Im} k_{5}\right)\right), \\
& m_{13}=\frac{1}{8} v^{2}\left(-\lambda_{1}(\sin 2 \beta+\sin 4 \beta)+\lambda_{2}(\sin 2 \beta-\sin 4 \beta)+\right. \\
& +2\left(\lambda_{3}+\lambda_{4}+\operatorname{Re} \lambda_{5}\right) \sin 4 \beta+4\left(\operatorname{Re} \lambda_{6}(\cos 2 \beta+\cos 4 \beta)+\right. \\
& \left.+\operatorname{Re} \lambda_{7}(\cos 2 \beta-\cos 4 \beta)\right), \\
& m_{14}=v\left(v_{3}\left(k_{1} \cos ^{2} \beta+k_{2} \sin ^{2} \beta\right)+\left(v_{3} \operatorname{Re} k_{3}+\frac{1}{\sqrt{2}} \operatorname{Re} k_{5}\right) \sin 2 \beta\right), \\
& m_{15}=v\left(v_{3} \operatorname{Im} k_{3}-\frac{1}{\sqrt{2}} \operatorname{Im} k_{5}\right) \sin 2 \beta, \\
& m_{22}=-\frac{1}{8}\left(v^{2}\left(2 \operatorname{Re} \lambda_{5} \sin 2 \beta+\operatorname{Re} \lambda_{6}(1+\cos 2 \beta)+\operatorname{Re} \lambda_{7}(1-\cos 2 \beta)\right)+\right. \\
& \left.+2 v_{3}\left(v_{3} \operatorname{Re} k_{3}+\sqrt{2} \operatorname{Re} k_{5}\right)\right) \csc \beta \sec \beta,
\end{aligned}
$$

$$
\begin{aligned}
& m_{23}= \frac{1}{2} v^{2}\left(\operatorname{Im} \lambda_{5} \cos 2 \beta+\left(\operatorname{Im} \lambda_{7}-\operatorname{Im} \lambda_{6}\right) \sin 2 \beta\right) \\
& m_{24}=-v\left(v_{3} \operatorname{Im} k_{3}+\frac{1}{\sqrt{2}} \operatorname{Im} k_{5}\right) \\
& m_{25}= v\left(v_{3} \operatorname{Re} k_{3}-\frac{1}{\sqrt{2}} \operatorname{Re} k_{5}\right) \\
& m_{33}=\frac{1}{16}\left(v ^ { 2 } \left(\lambda_{1}(1-\cos 4 \beta)+\lambda_{2}(1-\cos 4 \beta)+\right.\right. \\
& \quad+2\left(\lambda_{3}+\lambda_{4}+\operatorname{Re} \lambda_{5}\right)(\cos 4 \beta-1)-4\left(\operatorname{Re} \lambda_{6}(\cot \beta+\sin 4 \beta)+\right. \\
&\left.\left.\quad \quad+\operatorname{Re} \lambda_{7}(\tan \beta-\sin 4 \beta)\right)-4 v_{3}\left(\operatorname{Re} k_{3} v_{3}+\sqrt{2} \operatorname{Re} k_{5}\right) \csc \beta \sec \beta\right),
\end{aligned}
$$$$
m_{34}=\frac{1}{2} v\left(v_{3}\left(k_{2}-k_{1}\right) \sin 2 \beta+2\left(v_{3} \operatorname{Re} k_{3}+\frac{1}{\sqrt{2}} \operatorname{Re} k_{5}\right) \cos 2 \beta\right),
$$$$
m_{35}=v \cos 2 \beta\left(v_{3} \operatorname{Im} k_{3}-\frac{1}{\sqrt{2}} \operatorname{Im} k_{5}\right),
$$$$
m_{44}=v_{3}^{2} k_{4}-\frac{1}{2 \sqrt{2}}\left(\frac{v^{2}}{v_{3}} \operatorname{Re} k_{5} \sin \beta \cos \beta+3 v_{3} \operatorname{Re} k_{6}\right),
$$ 


$$
\begin{aligned}
& m_{45}=v^{2} \operatorname{Im} k_{3} \sin \beta \cos \beta-3 \sqrt{2} v_{3} \operatorname{Im} k_{6}, \\
& m_{55}=-\frac{1}{4 v_{3}}\left(v^{2}\left(4 \operatorname{Re} k_{3} v_{3}+\sqrt{2} \operatorname{Re} k_{5}\right) \sin \beta \cos \beta+9 \sqrt{2} v_{3}^{2} \operatorname{Re} k_{6}\right) .
\end{aligned}
$$

Заключительные замечания. В работе рассмотрены массовые состояния модели НМССМ и вычислены параметры эффективного потенциала в однопетлевом приближении с учетом вкладов скалярных суперсимметричных частиц. Вычислены массы нейтральных $C P$-чётных и $C P$-нечётных бозонов Хиггса.

Разработана программа в среде Mathematica для расчёта собственных состояний массовой матрицы бозонов Хиггса в НMCCM (с учётом диагонализации в локальном минимуме). Возможен учёт поправок во все параметры эффективного потенциала, в том числе включая нарушение $C P$-инвариантности комплексными параметрами. Расчёт проводится с произвольной $5 \times 5$-матрицей, проверки с предыдущими методами вычислений по $3 \times 3$-матрице совпадают.

Работа выполнена при финансовой поддержке грантом РФФИ 10-02-00525-а и частично в рамках госзаказа СамГУ 1.909.2011 и Соглашения 14.В37.21.1299 ФЦП. Э.Р. и Т.В. выражают благодарность за поддержку грантами 12-02-31795 КИАС РФФИ, Фонду Династия и МЦФФМ.

Авторы выражают признательность и благодарность В. П. Радченко и Оргкомитету Третьей международной конференции по математической физике и её приложениям за подготовку к изданию докладов и материалов конференции.

\section{БИБЛИОГРАФИЧЕСКИЙ СПИСОК}

1. H. P. Nilles, M. Srednicki, D. Wyler, "Weak interaction breakdown induced by supergravity" // Phys. Lett. B, 1983. Vol. 120, no. 4-6. Pp. 346-348.

2. J. M. Frère, D. R. T. Jones, S. Raby, "Fermion masses and induction of the weak scale by supergravity" // Nucl. Phys. B, 1983. Vol. 222, no. 1. Pp. 11-19.

3. J. P. Derendinger, C. A. Savoy, "Quantum effects and $\mathrm{SU}(2) \times \mathrm{U}(1)$ breaking in supergravity gauge theories" // Nucl. Phys. B, 1984. Vol.237, no. 2. Pp. 307-328.

4. J. R. Ellis, J. F. Gunion, H. E. Haber, L. Roszkowski, F. Zwirner, "Higgs bosons in a nonminimal supersymmetric model" // Phys. Rev. D, 1989. Vol. 39, no. 3. Pp. 844-869.

5. M. Drees, "Supersymmetric models with extended Higgs sector" // Int. J. Mod. Phys. A, 1989. Vol. 4, no. 14. Pp. 3635-3651.

6. U. Ellwanger, M. Rausch de Traubenberg, C. A. Savoy, "Particle spectrum in supersymmetric models with a gauge singlet" // Phys. Lett. B, 1993. Vol.315, no.3-4. Pp. 331-337, arXiv: hep-ph/9307322.

7. U. Ellwanger, M. Rausch de Traubenberg, C. A. Savoy, "Phenomenology of supersymmetric models with a singlet" // Nucl. Phys. B, 1997. Vol.492, no. 1. Pp. 21-50, arXiv: hep-ph/9611251.

8. S. F. King, P. L. White, "Resolving the constrained minimal and next-to-minimal supersymmetric standard models" // Phys. Rev. D, 1995. Vol.52, no. 7. Pp. 4183-4216, arXiv: hep-ph/9505326.

9. J. E. Kim, H. P. Nilles, "The $\mu$-problem and the strong CP-problem" // Phys. Lett. B, 1984. Vol. 138, no. 1-3. Pp. 150-154.

10. G. F. Giudice, A. Masiero, "A natural solution to the $\mu$-problem in supergravity theories" // Phys. Lett. B, 1988. Vol. 206, no. 3. Pp. 480-484.

11. J. E. Kim, H. P. Nilles, "Gaugino condensation and the cosmological implications of the hidden sector" // Phys. Lett. B, 1991. Vol. 263, no. 1. Pp. 79-85. 
12. E. J. Chun, J. E. Kim, H. P. Nilles, "A natural solution of the $\mu$ problem with a composite axion in the hidden sector" // Nucl. Phys. B, 1992. Vol.370, no. 1. Pp. 105-122.

13. J.A. Casas and C. Muñoz, "A natural solution to the $\mu$ problem" // Phys. Lett. B, 1993. Vol. 306, no. 3-4. Pp. 288-294, arXiv: hep-ph/9302227.

14. G. Lopes Cardoso, D. Lüst, T. Mohaupt, "Moduli spaces and target space duality symmetries in $(0,2) Z_{N}$ orbifold theories with continuous Wilson lines" // Nucl. Phys. B, 1994. Vol. 432, no. 1-2. Pp. 68-108, arXiv: hep-th/9405002.

15. I. Antoniadis, E. Gava, K. S. Narain, T. R. Taylor, "Effective $\mu$-term in superstring theory" // Nucl. Phys. B, 1994. Vol. 432, no.1-2. Pp. 187-204, arXiv: hep-th/9405024.

16. A. Brignole, L. E. Ibáñez, C. Muñoz, "Orbifold-induced $\mu$ term and electroweak symmetry breaking" // Phys. Lett. B, 1996. Vol.387, no. 4. Pp. 769-774, arXiv: hep-ph/9607405.

17. K. Choi, J. S. Lee, C. Muñoz, "Supergravity radiative effects on soft terms and the $\mu$ term" // Phys. Rev. Lett., 1998. no. 17. Pp. 3686-3689, arXiv: hep-ph/9709250.

18. R. D. Peccei, H. R. Quinn, "CP conservation in the presence of pseudoparticles" // Phys. Rev. Lett., 1977. no. 25. Pp. 1440-1443.

19. M. Bastero-Gil, C. Hugonie, S. F. King, D. P. Roy, S. Vempati, "Does LEP prefer the NMSSM?" // Phys. Lett. B, 2000. Vol.489, no. 3-4. Pp. 359-366, arXiv: hep-ph/0006198.

20. S. A. Abel, S. Sarkar, P. L. White, "On the cosmological domain wall problem for the minimally extended supersymmetric standard model" // Nucl. Phys. B, 1995. Vol. 454, no. 3. Pp. 663-681, arXiv: hep-ph/9506359.

21. C. Panagiotakopoulos, K. Tamvakis, "Stabilized NMSSM without domain walls" // Phys. Lett. B, 1999. Vol. 446, no. 3-4. Pp. 224-227, arXiv: hep-ph/9809475.

22. S. A. Abel, "Destabilising divergences in the NMSSM" // Nucl. Phys. B, 1996. Vol.480, no. 1-2. Pp. 55-72, arXiv: hep-ph/9609323.

23. J. Bagger, E. Poppitz, L. Randall, "Destabilizing divergences in supergravity theories at two loops" // Nucl. Phys. B, 1995. Vol. 455, no.1-2. Pp. 59-82, arXiv: hep-ph/9505244.

24. J. Bagger, E. Poppitz, "Destabilizing divergences in supergravity coupled supersymmetric theories" // Phys. Rev. Lett., 1993. Vol. 71, no. 15. Pp. 2380-2382, arXiv: hep-ph/9307317.

25. H. P. Nilles, M. Srednicki, D. Wyler, "Constraints on the stability of mass hierarchies in supergravity" // Phys. Lett. B, 1983. Vol.124, no. 5. Pp. 337-340.

26. U. Ellwanger, "Nonrenormalizable interactions from supergravity, quantum corrections and effective low-energy theories" // Phys. Lett. B, 1983. Vol.133, no. 3-4. Pp. 187-191.

27. U. Ellwanger, C. Hugonie, "Topologies of the (M+1)SSM with a singlino LSP at LEP2" // Eur. Phys. J. C, 2000. Vol. 13, no. 4. Pp. 681-690, arXiv: hep-ph/9812427.

28. U. Ellwanger, C. Hugonie, "Masses and couplings of the lightest Higgs bosons in the (M + 1)SSM" // Eur. Phys. J. C, 2002. Vol. 25, no. 2. Pp. 297-305, arXiv: hep-ph/9909260.

29. U. Ellwanger, J. F. Gunion, C. Hugonie, "NMHDECAY: A Fortran Code for the Higgs Masses, Couplings and Decay Widths in the NMSSM" // JHEP, 2005. Vol. 2005, no. 02, 066, arXiv: hep-ph/0406215.

30. U. Ellwanger, J. F. Gunion, C. Hugonie, S. Moretti, NMSSM Higgs discovery at the LHC, arXiv: hep-ph/0401228.

31. J. Ellis, J. F. Gunion, H. E. Haber, L. Roszkowski, F. Zwirner, "Higgs bosons in a nonminimal supersymmetric model" // Phys. Rev. D, 1989. Vol. 39, no. 3. Pp. 844-869.

32. Э. Н. Ахметзянова, М. В. Долгополов, М. Н. Дубинин, "Бозоны Хиггса в двухдублетной модели с нарушением СР-инвариантности" // ЯФ, 2005. Т. 68, № 11. С. 1913-1927; англ. пер.: E. N. Akhmetzyanova, M. V. Dolgopolov, M. N. Dubinin, "Higgs bosons in the two-doublet model involving CP violation" // Phys. Atomic Nuclei, 2005. Vol.68, no. 11. Pp. 1851-1865.

33. Э. Н. Ахметзянова, М. В. Долгополов, М. Н. Дубинин, "Нарушение СР-инвариантности в двухдублетном хиггсовском секторе МССМ" // ЭЧАЯ, 2006. Т. 37, № 5. С. 12851382; англ. пер.: E. N. Akhmetzyanova, M. V. Dolgopolov, M. N. Dubinin, "Violation of CP invariance in the two-doublet higgs sector of the MSSM" // Phys. Part. Nuclei, 2006. Vol. 37, no. 5. Pp. 677-734. 
34. E. N. Akhmetzyanova, M. V. Dolgopolov, M. N. Dubinin,, "Higgs bosons in the two-doublet model with CP violation" // Phys. Rev. D, 2005. Vol.71, no.7, 075008. 24 pp., arXiv: hep-ph/0405264.

35. M. Dolgopolov, M. Dubinin, I. Erofeev, E. Rykova, Threshold corrections to the MSSM effective Higgs potential: gaugino and higgsino contributions: PoS (QFTHEP2011) 068.

36. M. Maniatis, "The next-to-minimal supersymmetric extension of the standard model reviewed" // Int. J. Mod. Phys. A, 2010. Vol. 25, no. 18n19. Pp. 3505-3602.

37. А. О. Борисов, М. В. Долгополов, М. Н. Дубинин, Э. Н. Рыкова, "Аналитические выражения для пороговых поправок к температурному потенциалу Хиггса МССМ" // ЯФ, 2009. Т. 72, № 1. С. 175-180; англ. пер.: A. O. Borisov, M. V. Dolgopolov, E. N. Rykova, M. N. Dubinin, "Analytic expressions for threshold corrections to the finite-temperature higgs potential in the minimal supersymmetric standard model" // Phys. Atomic Nuclei, 2009. Vol. 72, no. 1. Pp. 167-172.

38. А. О. Борисов, М. В. Долгополов, "Однопетлевые поправки перенормировки поля в скалярном секторе МССМ" // ЯФ, 2010. Т. 73, №6. С. 1130-1133; англ. пер.: A. O. Borisov, M. V. Dolgopolov, "One-loop wave-function renormalization corrections in the scalar sector of the Minimal Supersymmetric Standard Model" // Phys. Atomic Nuclei, 2010. Vol.73, no. 6. Pp. 1093-1096.

39. M. Dolgopolov, M. Dubinin, E. Rykova, "Threshold corrections to the MSSM finite-temperature Higgs potential" // J. Mod. Phys., 2011. Vol. 2. Pp. 301-322, arXiv: 0901.0524 [hep-ph].

Поступила в редакцию 26/XI/2012;

в окончательном варианте - 01/II/2013.

MSC: 81V35; $81 \mathrm{T60}$

\section{EFFECTIVE HIGGS POTENTIAL IN NEXT-TO-MINIMAL SUPERSYMMETRIC STANDARD MODEL}

\section{T. V. Volkova ${ }^{1}$, M. V. Dolgopolov ${ }^{1}$, M. N. Dubinin ${ }^{2}$, E. N. Rykova ${ }^{1}$}

1 Samara State University,

1, Academician Pavlov st., Samara, 443011, Russia

2 Skobeltsyn Institute of Nuclear Physics,

Lomonosov Moscow State University,

Vorob'evy gory, Moscow, 119991, Russia.

E-mails: milandiya@yandex.ru, mikhaildolgopolov@rambler.ru, dubinin@theory.sinp.msu.ru, elzarykova@rambler.ru

Higgs sector of the Next-to-Minimal Sypersymmetric Model with CP violation in superpotential and in in the soft supersymmetry breaking sector is considered. One-loop corrections to effective potential parameters were calculated and incorporated to the evaluation of neutral Higgs bosons masses.

Key words: Higgs sector, nonminimal supersymmetric model, Higgs bosons masses, effective Higgs potential.

Original article submitted 26/XI/2012; revision submitted $01 / \mathrm{II} / 2013$.

Tatiana V. Volkova, Magistrant, Lab. of Mathematical Physics.

Mikhail V. Dolgopolov (Ph. D. (Phys. \& Math.)), Associate Professor, Dept. of General \& Theoretical Physics. Mikhail N. Dubinin (Dr. Sci. (Phys. \& Math.)), Leading Researcher, Division of Theoretical High Energy Physics. Elza N. Rykova (Ph. D. (Phys. \& Math.)), Senior Lecturer, Dept. of General \& Theoretical Physics. 